\title{
FINITELY GENERATED COMMUTATIVE SEMIGROUPS
}

\author{
by D. B. MCALISTER and L. O'CARROLL
}

(Received 17 January, 1969; revised 11 September, 1969)

Since all the semigroups considered in this paper are commutative, we shall use the terms "semigroup" and "group" where we actually mean "commutative semigroup" and "commutative group". Some basic results from the theory of semigroups are required and will be used without explicit mention; these results may be found in $[1, \S 4.3]$. We shall denote the additive semigroups of integers, positive integers, negative integers, positive rationals by $\mathbf{Z}, \mathbf{Z}^{+}, \mathbf{Z}^{-}, \mathbf{Q}^{+}$respectively.

1. Introduction. In his book The theory of finitely generated commutative semigroups, L. Rédei has carried out a deep analysis of the congruences on finitely generated free semigroups. As a consequence of this theory, one can, in principle, construct all finitely generated semigroups. However, Rédei's theory does not give rise to a structure theorem for such semigroups. In this paper we consider the problem of giving a description of finitely generated semigroups in the spirit of the so-called "Fundamental theorem of finitely generated groups " [3, Theorems 10.3, 10.4]. We shall state this result as Theorem 1.1 because it serves as a model for our results; taken with our results, it also shows where the analogy between groups and semigroups breaks down.

THEOREM 1.1. Let $G$ be a finitely generated group.

(I) $G \approx \mathbf{Z}^{n} \times F$ for some non-negative integer $n$ and some finite group $F$. The integer $n$ is unique and the group $F$ is unique up to isomorphism.

(II) $F$ is a direct product of cyclic groups of prime power order.

(III) Each subgroup of $G$ is finitely generated.

Any finitely generated semigroup can be uniquely expressed as the union of a semilattice of archimedean semigroups. Hence the structure of finitely generated semigroups depends on the structure of the archimedean subsemigroups of finitely generated semigroups. Examples 1.2 and 1.3 show that these subsemigroups need not themselves be finitely generated so that we cannot say much, in general, about the archimedean components of finitely generated semigroups. ([10], [11] give a construction for all archimedean semigroups, but this is very complicated). Indeed, we have not been able to characterise those semigroups which are archimedean components of finitely generated semigroups although some results in this direction are given in Proposition 4.4 and Theorem 6.2.

EXAMPLE 1.2. Let $S=F(a, b)$ be the free semigroup on two generators $a, b$. Then $S$ has three archimedean classes $\langle a\rangle,\langle b\rangle$ and $K=\left\{a^{m} b^{n}: m, n \in \mathbf{Z}^{+}\right\}$. Since $K \mid K^{2}=$ $\left\{a b^{n}, a^{n} b: n \in \mathbf{Z}^{+}\right\}$is infinite, $K$ is not finitely generated.

EXAMPLE 1.3. Let $S=F\left(a, b ; a^{2} b^{2}=b^{2}\right)$. Then $S$ has two archimedean classes $\langle a\rangle$ 
and $K=\left\{b^{n}, a^{n} b, a b^{n}: n \in \mathbf{Z}^{+}\right\}$. Since $K \backslash K^{2}=\left\{b, a^{n} b: n \in \mathbf{Z}^{+}\right\}$is infinite, $K$ is not finitely generated.

Proposition 1.4 sums up what we can usefully say, in general, about the archimedean components of finitely generated semigroups.

PROPOSITION 1.4. If $S$ is a finitely generated semigroup, then $S$ is a finite semilatice of archimedean semigroups. Each maximal archimedean class and each subgroup of $S$ is finitely generated.

Proof. Since $S$ is finitely generated, so is its maximal semilattice homomorphic image $\Lambda$; because each element of $\Lambda$ is idempotent, this implies that $\Lambda$ is finite. Hence $S$ is the union of the finite semilattice $\Lambda$ of archimedean semigroups.

Each maximal archimedean class of $S$ is clearly generated by those generators of $S$ which it contains and hence is finitely generated. It thus remains to prove that each subgroup of $S$ is finitely generated. In fact, by Theorem 1.1, (III), it suffices to show that each maximal subgroup of $S$ is finitely generated.

Let $H$ be a maximal subgroup of $S$; then $H$ is contained in some archimedean class $S_{\alpha}$ of $S$. Let $D=\bigcup\left\{S_{\beta}: \beta \geqq \alpha\right\}$ and denote by $e$ the identity of $H$. Then

$$
S_{\alpha} e \subseteq D e=(D e) e \subseteq S_{\alpha} e,
$$

so that $D e=S_{x} e$. But, because $H$ is a maximal subgroup of the archimedean semigroup $S_{\alpha}, H=S_{\alpha} e$; hence $H=D e$. It follows that we can define a mapping $\theta: S \rightarrow H^{\circ}$ by

$$
x \theta=\left\{\begin{array}{cl}
x e & \text { if } x \in D, \\
0 & \text { otherwise }
\end{array}\right.
$$

Since $D$ is the complement of a prime ideal of $S[6$, Lemma 1.3], it is clear that $\theta$ is a homomorphism of $S$ onto $H^{0}$; thus $H^{0}$ is finitely generated. But $H^{0}$ has maximal archimedean class $H$ so that, by the second part of the proposition, $H$ is finitely generated.

COROLLARY. A finitely generated semigroup is regular (i.e., is a union of groups) if and only if it is a finite semilattice of finitely generated groups.

Although we cannot say much about the non-finitely generated archimedean subsemigroups of a finitely generated semigroup, finitely generated archimedean semigroups with and without idempotents can be described in some detail; see Theorem 3.10 and Proposition 4.2. Because of this, we shall consider semigroups which obey the ascending chain condition on subsemigroups (A.C.C. for short). The following proposition follows by a standard argument.

Proposition 1.5. A semigroup obeys the A.C.C. if and only if each subsemigroup is finitely generated.

COROLlary. A semigroup obeys the A.C.C. if and only if it is a finite semilattice of archimedean semigroups each of which obeys the A.C.C.

As a consequence of this corollary, the problem of determining the structure of all semigroups which obey the A.C.C. reduces to that of finding those archimedean semigroups which obey the A.C.C. The congruences which we use to investigate the latter problem are dis- 
cussed in the next section and the problem itself is tackled in the third and fourth sections.

Separative semigroups which obey the A.C.C. are neatly characterised by Theorem 1.6; the proof is given in $\S 5$.

THEOREM 1.6. Let $S$ be a separative semigroup. Then $S$ obeys the A.C.C. if and only if it can be embedded in a finite semilattice of groups $\mathbf{Z} \times F_{\alpha}$, where each $F_{\alpha}$ is a finite group.

\section{Some important congruences.}

Defintrion 2.1. [1] Let $S$ be a semigroup; then

$$
\sigma=\left\{(a, b) \in S \times S: a^{n} b=a^{n+1} ; a b^{n}=b^{n+1} \text { for some } n \in Z^{+}\right\} .
$$

Proposition 2.2. [1] $\sigma$ is the finest congruence $\rho$ on $S$ such that $S / \rho$ is separative. If $S$ is archimedean, then $S / \sigma$ is cancellative; in particular, a separative archimedean semigroup is cancellative. $a=b$.

Definition 2.3. [6] A semigroup $S$ is torsion free if, for $a, b \in S, n \in \mathbf{Z}^{+}, a^{n}=b^{n}$ implies

Proposition 2.4. [6] Let $S$ be a semigroup and let

$$
\tau=\left\{(a, b) \in S \times S: a^{n}=b^{n} \text { for some } n \in Z^{+}\right\} .
$$

Then $\tau$ is the finest congruence $\rho$ on $S$ such that $S / \rho$ is torsion free. Further $\sigma \subseteq \tau$.

COROLlary 1. Let $S$ be a semigroup. Then the following are equivalent:

(a) $S$ has an idempotent;

(b) $S / \sigma$ has an idempotent;

(c) $S / \tau$ has an idempotent.

If, further, $S$ is archimedean, then $(a),(b),(c)$ are equivalent to

(d) there exist $x, y \in S$ with $x=x y$.

Proof. Clearly $(a)$ implies $(b)$ and $(b)$ implies $(c)$. Hence, to prove the equivalence of these three statements, it remains to show that $(c)$ implies $(a)$. Suppose that $(c)$ is satisfied and let $x \in S$ be such that $\left(x^{2}, x\right) \in \tau$. Then $x^{n}=\left(x^{2}\right)^{n}=\left(x^{n}\right)^{2}$ for some $n \in \mathbf{Z}^{+}$; hence $x^{n}$ is idempotent.

In any semigroup with an idempotent $e$, there exist $x(=e), y(=e)$ such that $x=x y$. Therefore $(a)$ implies $(d)$, without any restriction on $S$. If $S$ is archimedean, then $S / \sigma$ is cancellative so that $x=x y$ gives $\left(y, y^{2}\right) \in \sigma$; that is, $S / \sigma$ has an idempotent. Hence $(d)$ implies (b).

COROLlaRY 2. If $S$ is an archimedean semigroup, then $S / \tau$ is cancellative.

Proof. $S / \tau$ is clearly separative. Hence, by Proposition $2.2, S / \tau$ is cancellative.

Definition 2.5. Let $S$ be a semigroup. Then, for each $a \in S$,

$$
\rho_{a}=\left\{(x, y) \in S \times S: x a^{n}=y a^{m} \text { for some } m, n \in Z^{+}\right\} \text {. }
$$




\section{FINITELY GENERATED COMMUTATIVE SEMIGROUPS}

$\rho_{a}$ is Dubreil's reversible congruence $P_{\langle a\rangle}$, where $\langle a\rangle$ is the semigroup of $S$ generated by a. Hence we have the following proposition.

Proposition 2.6 [2], [9]. Let $S$ be a semigroup. Then, for each $a \in S, \rho_{a}$ is the finest congruence $\rho$ on $S$ such that $S / \rho$ has identity a $\rho^{\natural}$.

If $S$ is archimedean, then $\rho_{a}$ is the finest congruence on $S$ such that $S / \rho$ is a group with identity $a \rho^{\natural}$.

COROLLARY. Let $S$ be a semigroup and let $S=\bigcup\left\{S_{\lambda}: \lambda \in \Lambda\right\}$ be the expression of $S$ as the union of a semilattice $\Lambda$ of archimedean semigroups $S_{\lambda}$. Suppose that $\Lambda$ has a minimum member $\alpha$. Then, for each $a \in S_{\alpha}, \rho_{a}$ is the finest congruence $\rho$ on $S$ such that $S / \rho$ is a group with identity $a \rho^{\natural}$.

If $\theta$ is a homomorphism of $S$ onto a group $G$, there exists $a \in S_{\alpha}$ such that $\rho_{a} \subseteq \theta \circ \theta^{-1}$.

Proof. A straightforward argument shows that $\rho_{a}$ is the finest group congruence $\rho$ on $S$ whose quotient has identity $a \rho^{\natural}$.

Suppose that $\theta$ is a homomorphism of $S$ onto a group $G$. Then, since $S_{\alpha}$ is an ideal of $S$, $S_{a} \theta$ is an ideal of $G$; since $G$ is a group, this means that $G=S_{\alpha} \theta$. In particular, there exists $a \in S_{a}$ such that $a \theta$ is the identity of $G$. By the first part of the proposition, $\rho_{a} \subseteq \theta \circ \theta^{-1}$ for this $a$.

Proposition 2.7. If $S$ is an archimedean semigroup without idempotents, then

for each $a \in S$.

$$
\sigma=\tau \cap \rho_{a}
$$

Proof. By Proposition 2.6, $S / \rho_{a}$ is a group and so is separative; hence $\sigma \subseteq \rho_{a}$. But, by Corollary 2 to Proposition 2.4, $\sigma \subseteq \tau$. Thus we have $\sigma \subseteq \rho_{a} \cap \tau$.

Conversely, let $(x, y) \in \rho_{a} \cap \tau$. Then $x^{n}=y^{n}$ and $x a^{r}=y a^{s}$ for some $n, r, s \in \mathbf{Z}^{+}$. These equations give $x^{n} a^{n}=x^{n} a^{s n}$ and, since $S$ has no idempotents, it follows that $r=s$. But then $x a^{\prime \prime}=y a^{\prime}$ and so, since $S / \sigma$ is cancellative, $(x, y) \in \sigma$. Hence $\rho_{a} \cap \tau \subseteq \sigma$.

Proposition 2.7 is useful for describing the structure of finitely generated archimedean semigroups which are cancellative. To characterise those that are not cancellative, we require another congruence.

Definition 2.8. Let $S$ be a semigroup. Then, for each $a \in S$,

$$
\mu_{a}=\Delta \cup\left\{(x, y) \in S \times S: x=a u, y=a v, a^{n} u=a^{m} v \text { for some } u, v \in S^{1}, m, n \in \mathbf{Z}^{+}\right\},
$$

where $\Delta$ is the identity congruence on $S$.

Proposirion 2.9. Let a be an element of a semigroup $S$. Then $\mu_{a}$ is the finest congruence on $S$ which contains $\left(a, a^{2}\right)$.

Proof. This is straightforward.

PROPOSITION 2.10. Let $S$ be an archimedean semigroup without idempotents. Then, for each $a \in S, \mu_{a} \cap \tau=\lambda_{a}$, where

$$
\lambda_{a}=\Delta \cup\left\{(x, y) \in S \times S: x=a u, y=a v, a^{n} u=a^{n} v \text { for some } u, v \in S^{1}, n \in \mathbf{Z}^{+}\right\} .
$$


Proof. Suppose that $(x, y) \in \lambda_{a}$ but $x \neq y$. Then, for some $u, v \in S^{1}, n \in \mathbf{Z}^{+}, x=a u, y=a v$ and $a^{n} u=a^{n} v$. Since $a^{n} u=a^{n} v$, we certainly have $\left(a^{n} u, a^{n} v\right) \in \tau$ but, by Corollary 2 to Proposition $2.4, S / \tau$ is cancellative so that this implies that $(a u, a v) \in \tau$; that is, that $(x, y) \in \tau$. Hence $\lambda_{a} \subseteq \tau$. Further, from the definitions of $\mu_{a}, \lambda_{a}$, it is clear that $\lambda_{a} \subseteq \mu_{a}$; it follows that $\lambda_{a} \subseteq \mu_{a} \cap \tau$.

Conversely, suppose that $(x, y) \in \mu_{a} \cap \tau$ and that $x \neq y$. Then, for some $u, v \in S^{\mathfrak{l}}, n, r, s \in \mathbf{Z}^{+}$,

$$
x^{n}=y^{n}, \quad x=a u, \quad y=a v \text { and } a^{r} u=a^{s} v .
$$

It follows, from the first three of these equations, that $a^{n} u^{n}=a^{n} v^{n}$ and therefore that $a^{r n} u^{n}=$ $a^{s n} v^{n}=a^{s n} u^{n}$. By hypothesis, $S$ is archimedean without idempotents; therefore, by Corollary $1(d)$ to Proposition $2.4, r=s$. Thus $(x, y) \in \lambda_{a}$. Since $(x, y) \in \lambda_{a}$ if $x=y$, this shows that $\mu_{a} \cap \tau=\lambda_{a}$ and so completes the proof of the proposition.

CoROllary. Let $S$ be an archimedean semigroup that has no idempotents. Suppose that $a \in S$ has the property that $a^{2} w=a^{2} z$ implies $a w=a z$ for $w, z \in S^{1}$. Then $\mu_{a} \cap \tau=\Delta$.

We shall use the congruences $\mu_{a}, \tau$ in the next section to study the structure of finitely generated archimedean semigroups that have no idempotents. As the final result of this section, we prove that these are almost the only congruences on such semigroups. The proof of this result requires the structure theory given in the next section. However this seems the best place to give the result.

Proposition 2.11. Let $S$ be a finitely generated archimedean semigroup without idempotents and let $\rho$ be a congruence on $S$. Then

(i) $S / \rho$ has an idempotent if and only if $p \notin \tau$;

(ii) if $\rho \subseteq \tau$, then $\rho=\tau \cap \varphi$, where $\varphi$ is a congruence such that $\varphi \nsubseteq \tau$.

Proof. (i) Since $S$ admits no idempotents, it follows, from Corollary 1 to Proposition 2.4, that $S / \tau$ is without idempotents. Hence, if $\rho \subseteq \tau, S / \rho$ does not have an idempotent.

Conversely, if $\rho \notin \tau$, there exists $(x, y) \in \rho$ such that $x^{n} \neq y^{n}$ for any $n \in \mathbf{Z}^{+}$. By Theorem 3.4, there exist $r, s \in \mathbf{Z}^{+}$such that $x^{r}=y^{s}$ and, by the preceding sentence, $r \neq s$. Since $(x, y) \in \rho$ and $x^{r}=y^{s}$ we have $\left(x^{r}, x^{s}\right) \in \rho$. Hence $S / \rho$ admits an idempotent.

(ii) In this case, $S / \rho$ is finitely generated and archimedean; further, since $\rho \subseteq \tau, S / \rho$ is without idempotents. Hence, by the corollary to Theorem 3.10 , there exists $a \in S$ such that $\left(a^{2} x, a^{2} y\right) \in \rho$ implies $(a x, a y) \in \rho$ for $x, y \in S^{1}$. Let

$$
\varphi=\rho \cup\left\{(x, y) \in S \times S:(x, a u) \in \rho,(y, a v) \in \rho,\left(a^{n} u, a^{m} v\right) \in \rho \text { for some } u, v \in S^{1}, m, n \in Z^{+}\right\} .
$$

Then it can be shown that $\varphi$ is a congruence on $S$ and an argument like that used in the proof of Proposition 2.10 shows that $\rho=\varphi \cap \tau$. Clearly $\mu_{a} \subseteq \varphi$ and so, since $\mu_{a} \ddagger \tau, \varphi \nsubseteq \tau$.

REMARK. If $\rho$ is a congruence on $S$ with $\rho \neq \tau$, then $S / \rho$ has an idempotent and so it follows, from Proposition 3.2 and Theorem 3.4, that $S / \rho$ is finite; in particular, $S / \mu_{a}$ is finite for each $a \in S$. Thus each congruence on $S$ is determined by $\tau$, some $\mu_{a}$ and a congruence on a finite semigroup. 


\section{Archimedean semigroups without idempotents.}

Definition 3.1 [6]. A semigroup is rational if, for each $a, b \in S$, there exist positive integers $m, n$ such that $a^{m}=b^{n}$.

The semigroups described in Definition 3.1 have also been called power joined (see for example [5]). In view of Theorem 3.5, we have preferred to call these semigroups rational. section.

The following simple consequence of Definition 3.1 will be used several times in this

Proposition 3.2. Let $S$ be a finitely generated semigroup. Then $S$ is rational with an idempotent if and only if it is a finite archimedean semigroup.

Proof. Suppose that $S$ is rational with an idempotent $e$ and let $x_{1}, \ldots, x_{n}$ be generators for $S$. Then, for each $i=1, \ldots, n$, there exists $m_{i} \in \mathbf{Z}^{+}$such that $x^{m_{1}}=e$. It follows that $S$ has less than $\left(m_{1}+1\right)\left(m_{2}+1\right) \ldots\left(m_{n}+1\right) 2^{n}$ elements; thus $S$ is finite.

Conversely, suppose that $S$ is a finite archimedean semigroup and let $a, b \in S$. Then there exist $m, n \in \mathbf{Z}^{+}$such that $a^{m}, b^{n}$ are idempotent. Since an archimedean semigroup can have at most one idempotent, it follows that $a^{m}=b^{n}$. Hence $S$ is rational.

Petrich [7] has proved the following result.

THEOREM 3.3. Let $S$ be a finitely generated archimedean semigroup that has no idempotents. If $S$ is cancellative, then $S$ is rational.

Proposition 2.4 allows us to generalise this theorem by dropping the assumption that $S$ is cancellative. This extension is of fundamental importance in this paper; we state it as Theorem 3.4. Theorem 3.4 has also been found by Tamura and Levin (see the appendix to [11] and also [5]).

THEOREM 3.4. A finitely generated archimedean semigroup without idempotents is a rational semigroup.

Proof. If $S$ is a finitely generated archimedean semigroup without idempotents, then, by Corollary 2 to Proposition $2.4, S / \tau$ is a finitely generated cancellative archimedean semigroup that has no idempotents; hence, by Theorem 3.3, $S / \tau$ is rational.

Let $a, b \in S$; then, since $S / \tau$ is rational, there exist $m, n \in \mathbf{Z}^{+}$such that $\left(a^{m}, b^{n}\right) \in \tau$. Thus there exists $r \in \mathbf{Z}^{+}$such that $a^{m r}=b^{n r}$. Hence $S$ is rational.

COROLLARY. Let $S$ be a finitely generated archimedean semigroup without idempotents. Then $S / \mu_{a}$ is finite for each $a \in S$.

The following theorem from [6] characterises rational semigroups in terms of their maximal torsion free homomorphic images.

THEOREM 3.5. Let $S$ be a semigroup without idempotents. Then $S$ is rational if and only if $S / \tau$ is isomorphic to a subsemigroup of $\mathrm{Q}^{+}$.

COROLLARY. A finitely generated semigroup $S$ is rational without idempotents if and only if $S / \tau$ is isomorphic to a subsemigroup of $\mathbf{Z}^{+}$. 
Proof. If $S / \tau$ is isomorphic to a subsemigroup of $\mathrm{Z}^{+}$, then, by Theorem $3.5, S$ is rational.

Conversely, if $S$ is rational without idempotents, then $S / \tau$ is isomorphic to a finitely generated subsemigroup of $\mathrm{Q}^{+}$. Let $p_{i} / q_{i}(1 \leqq i \leqq n)$ be generators for $S / \tau$ where, for each $i$, $p_{i}, q_{i} \in \mathbf{Z}^{+}$. Clearly the mapping $\theta: x \rightarrow x q_{1} \ldots q_{n}$ is an isomorphism of $S / \tau$ onto a subsemigroup of $\mathbf{Z}^{+}$.

We are now in a position to state a structure theorem for rational semigroups. This theorem improves the result, stated without proof at the end of [6], by doing away with the factor sets.

THEOREM 3.6. Let $S$ be a separative semigroup without idempotents. Then $S$ is rational if and only if $S$ is isomorphic to a subsemigroup of $G \times \mathbf{Q}^{+}$for some periodic group $G$.

Proof. If $S$ is isomorphic to a subsemigroup of $G \times \mathbf{Q}^{+}$, then, clearly, $S$ is rational and cancellative; further it has no idempotents.

Conversely, if $S$ rational, it follows from Proposition 2.7 that $\rho_{a} \cap \tau=\Delta$ for each $a \in S$. Hence $S$ is isomorphic to a subsemigroup of $S / \rho_{a} \times S / \tau$. Since $S$ is rational, $G=S / \rho_{a}$ is a rational, and hence periodic, group. Further, by Theorem $3.5, S / \tau$ is isomorphic to a subsemigroup of $\mathbf{Q}^{+}$. Hence $S$ is isomorphic to a subsemigroup of $G \times \mathbf{Q}^{+}$.

A further consequence of Proposition 2.7 is that any finitely generated rational semigroup is embeddable in $\mathbf{Z}^{+} \times G$ for some finite group $G$. To prove the converse of this and some stronger results, we need deeper theory.

Proposition 3.7. Let $S$ be a rational semigroup that has no idempotents. Then the following are equivalent:

(i) $S$ has a cancellative ideal;

(ii) there exists $a \in S$ such that $a^{2} x=a^{2} y$ implies $a x=a y$ for $x, y \in S^{1}$;

(iii) $S$ is isomorphic to a subsemigroup of $P \times Q^{+}$for some periodic semigroup $P$ that has a unique idempotent.

Proof. (i) $\Rightarrow$ (ii). Suppose that $S$ has a cancellative ideal $C$ and pick $a \in C$. Then, for any $x, y \in S^{1}, a x, a y \in C$. Hence, since $C$ is cancellative, $a^{2} x=a^{2} y$ implies $a x=a y$.

(ii) $\Rightarrow$ (iii). If $a \in S$ has the cancellation property described in (ii), then, by the Corollary to Proposition 2.10, $\mu_{a} \cap \tau=\Delta$. It follows that $S$ is isomorphic to a subsemigroup of $S / \mu_{a} \times S / \tau$. By Theorem 3.5, $S / \tau$ is isomorphic to a subsemigroup of $\mathrm{Q}^{+}$while $S / \mu_{a}$ is rational with an idempotent. But it is easy to see that a rational semigroup that admits an idempotent is periodic with a unique idempotent. Hence (iii) holds.

(iii) $\Rightarrow$ (i). Suppose that $S \subseteq \mathrm{Q}^{+} \times P$, where $P$ is periodic and has only one idempotent and let $K=S \cap\left(\mathrm{Q}^{+} \times \operatorname{Ker} P\right)$. If $a=(r, p) \in S$, then, for some $n \in \mathbf{Z}^{+}$, $p^{n}$ belongs to $\operatorname{Ker} P$; hence $a^{n}=\left(n r, p^{n}\right) \in S \cap\left(Q^{+} \times \operatorname{Ker} P\right)$ so that $K$ is non-void. Since $\mathbf{Q}^{+}$is cancellative and $\operatorname{Ker} P$ is a cancellative (in fact, a group) ideal of $P, K$ is clearly a cancellative ideal of $S$. Hence (iii) implies (i).

EXAMPLe 3.8. Let $K$ be as in Example 1.3. Then $K$ is isomorphic to a subsemigroup of $\mathrm{Z}^{+} \times P$, where $P=\left\{x_{n}: n \geqq 1\right\} \cup\{e, f\}$ and 


$$
\begin{array}{rlrl}
x_{m} x_{n} & =\left\{\begin{array}{l}
f \text { if } m+n \text { is odd } \\
e \text { if } m+n \text { is even }
\end{array}\right\}, & e^{2}=f^{2}=e, e f=f e=f, \\
x_{m} f=\left\{\begin{array}{ll}
f \text { if } m \text { is even } \\
e \text { if } m \text { is odd }
\end{array}\right\}, & x_{m} e=\left\{\begin{array}{l}
f \text { if } m \text { is odd } \\
e \text { if } m \text { is even }
\end{array}\right\} .
\end{array}
$$

Proposition 3.7 gives a partial characterisation of rational semigroups. The next theorem shows that this characterisation applies to all finitely generated rational semigroups that are without idempotents. To prove the theorem we require the following lemma; the first part of the lemma is due to Tamura [9].

Lemma 3.9. Let $S$ be an archimedean semigroup that has no idempotents. Pick $a \in S$ and let $T_{n}=S a^{n} \backslash S a^{n+1}$ for each non-negative integer $n$, where $x a^{0}=x$ for each $x \in S$. Then

(i) $S=\bigcup\left\{T_{n}: n \geqq 0\right\}$;

(ii) each $T_{n}$ is non-void;

(iii) if $m \geqq n$, then $T_{m} \subseteq T_{n} a^{m-n}$.

Proof. (i) Tamura [9] has shown that $\bigcap\left\{S a^{n}: n \geqq 0\right\}=\square$. Hence, for each $x \in S$, there exists $n \geqq 0$ such that $x \in S a^{n} \backslash S a^{n+1}=T_{n}$. It follows that $S=\bigcup\left\{T_{n}: n \geqq 0\right\}$.

(ii) Since $S$ does not have an idempotent, it follows, from Corollary 1 to Proposition 2.4, that $a^{n+1} \notin S a^{n+1}$. Hence $a^{n+1} \in T_{n}$.

(iii) If $x \in T_{m}$, then $x=a^{m} u$ for some $u \in S$. Put $y=a^{n} u$; then $y \in S a^{n}$ and $x=y a^{m-n}$. Since $x \notin S a^{m+1}, y$ cannot belong to $S a^{n+1}$. Hence $y \in T_{n}$. This holds for any $x \in T_{m}$; so we have $T_{m} \subseteq T_{n} a^{m-n}$.

COROLlary. Let $S$ be an archimedean semigroup that has no idempotents. Then $S$ is generated by $S \backslash S a$ for each $a \in S$.

Proof. By (i) and (iii) of Lemma 3.9, $S=\bigcup\left\{T_{0} a^{n}: n \geqq 0\right\}$. Since $S$ has no idempotents, $a \in T_{0}$. Hence $S$ is generated by $T_{0}=S \backslash S a$.

REMARK. If $S$ is a finitely generated archimedean semigroup without idempotents, then it can be shown that $S$ is generated by $S \backslash S^{2}$. As we do not require this result, we omit a proof.

TheOREM 3.10. Let $S$ be a finitely generated semigroup. Then $S$ is archimedean without idempotents if and only if it is isomorphic to a subsemigroup of $\mathrm{Z}^{+} \times F$ for some finite semigroup $F$ that has a unique idempotent.

Proof. If $F$ is a finite semigroup that has a unique idempotent, then, by Proposition 3.7, $\mathrm{Z}^{+} \times F$ is rational. Hence every subsemigroup of $Z^{+} \times F$ is rational.

Conversely, suppose that $S$ is archimedean and has no idempotents; then $S$ is rational. We shall show that there exists $a \in S$ which satisfies (ii) of Proposition 3.7. Then, as in the proof of that proposition, $S$ is isomorphic to a subsemigroup of $S / \tau \times S / \mu_{a}$. By the corollary to Theorem 3.5, $S / \tau$ is isomorphic to a subsemigroup of $\mathbf{Z}^{+}$while, by the corollary to Theorem $3.4, F=S / \mu_{a}$ is finite with a unique idempotent. Hence $S$ is isomorphic to a subsemigroup of $\mathbf{Z}^{+} \times F$. 
To demonstrate the existence of such an $a$, we proceed as follows. Pick $b \in S$ and, for each non-negative integer $n$, let $T_{n}=S b^{n} \backslash S b^{n+1}$. Then, by Lemma 3.9, each $T_{n}$ is non-void and $\left|T_{m}\right| \leqq\left|T_{n}\right|$ if $m \geqq n$. Hence

$$
\left|T_{0}\right| \geqq\left|T_{1}\right| \geqq \ldots \geqq\left|T_{n}\right| \geqq \ldots>0 .
$$

Now $\left|T_{0}\right|=|S| S b|\leqq| S / S b \mid$, where $S / S b$ is a finitely generated nilpotent semigroup; since a finitely generated nilpotent semigroup is finite, it follows that $T_{0}$ is finite. Hence there exists $N \geqq 0$ such that $\left|T_{n}\right|=\left|T_{N}\right|$ for all $n \geqq N$. Suppose that $T_{N}=\left\{t_{1}, \ldots, t_{r}\right\}$; then, for each $n \geqq N$,

$$
T_{n}=\left\{t_{1} b^{n-N}, \ldots, t_{r} b^{n-N}\right\}
$$

where these elements are distinct.

Let $a=b^{N}$ and consider $x, y \in S^{1}$ such that $a^{2} x=a^{2} y$. Then $a x=t_{i} b^{u}, a y=t_{j} b^{v}$ for some $u, v \geqq 0$. Since $a^{2} x=a^{2} y$, this means that $t_{i} b^{u+N}=t_{j} b^{v+N}$. Hence, since distinct $T_{i}$ are disjoint, it follows from (1) that $u=v$. But then the distinctness of elements in (1) gives $t_{i}=t_{j}$. Hence $a x=a y$.

COROllary. Let $S$ be a finitely generated archimedean semigroup and let $b \in S$. Then there exists $a \in\langle b\rangle$ such that, for all $x, y \in S^{1}, a^{2} x=a^{2} y$ implies $a x=a y$.

Proof. If $S$ has an idempotent, then some power of $b$ belongs to $\operatorname{Ker} S$ which is a group. This power of $b$ satisfies the conclusions of the corollary.

If $S$ has no idempotent, this is the major part of the proof of Theorem 3.10.

REMARK. If $F$ is a finite semigroup, then $\mathrm{Z}^{+} \times F$ is not finitely generated unless $F^{2}=F$. Thus it follows, from Corollary 2 to Theorem 4.1, that, under the conditions of Theorem 3.10, $\mathbf{Z}^{+} \times F$ is finitely generated if and only if $F$ is a finite group.

It is an easy deduction from Theorem 3.10 that a finitely generated archimedean semigroup $S$ with no idempotents has a maximal cancellative ideal $K$. The canonical homomorphism $\sigma^{\natural}: S \rightarrow S / \sigma$ embeds $K$ naturally in $S / \sigma$. In general, this embedding is proper (see Example 3.11). However, if $S$ has an idempotent $e$, then $S e$ is the maximal cancellative ideal of $S$. In fact, $S e$ is the group kernel of $S$ and the maximal separative homomorphic image of $S$.

EXAMPLE 3.11. Let $S=F\left(a, b ; a b^{2}=b^{3}, b a^{2}=a^{3}\right)$. Then $a^{2} b^{2}=a^{4}=b^{4}$ and $S=$ $\langle b\rangle \cup\left\{a, a^{2}, a^{3}, a b\right\}$, so that $S$ is a finitely generated archimedean semigroup without idempotents. $K=\left\{b^{n}: n \geqq 2\right\}$ is a maximal cancellative ideal of $S$ but $K \sigma^{\natural}$ is a proper ideal of $S / \sigma ;\left(b, b^{n}\right) \notin \sigma$ for any $n \geqq 2$, since otherwise $S / \sigma$ would have an idempotent, which would contradict Proposition 2.4, Corollary 1.

There is a strong analogy between Part (I) of Theorem 1.1 and the structure, of finitely generated archimedean semigroups without idempotents, described in Theorem 3.10. Our next theorem shows that the analogue of Part (III) of Theorem 1.1 also holds for such semigroups.

THEOREM 3.12. Let $S$ be an archimedean semigroup that has no idempotents. Then $S$ obeys the A.C.C. if and only if it is finitely generated. 
Proof. If $S$ obeys the A.C.C., it is certainly finitely generated. Hence it remains to prove the converse implication. To do this, we show that every subsemigroup of $S$ is finitely generated.

Let $K$ be a subsemigroup of $S$. Then, by the corollary to Theorem 3.10, there exists $a \in K$ such that $a^{2} x=a^{2} y$ implies $a x=a y$ for $x, y \in S^{1}$. Suppose that, for some $x, y \in K \backslash K a$ $(x, y) \in \mu_{a}$ but $x \neq y$. Then there exist $u, v \in S^{1}$, with $u \neq v$, such that $x=a u, y=a v$ and $a^{n} u=a^{m} v$ for some $m, n \in \mathbf{Z}^{+}$. If, for example, $m>n$, then $a^{n} u=a^{n} v a^{m-n}$ and so, because of the cancellation properties of $a, a u=a v a^{m-n}$ which gives $x=y a^{m-n}$. This contradicts the hypothesis that $x, y \in K \backslash K a$. If $m=n$, then, by the cancellation properties of $a$, we get $a u=a v$; that is, $x=y$. Hence distinct elements of $K \backslash K a$ are distinct modulo $\mu_{a}$. By the corollary to Theorem 3.4, $S / \mu_{a}$ is finite; thus $K \backslash K a$ is finite.

Since $K$ is a subsemigroup of $S$ (which is rational without idempotents) $K$ is archimedean and has no idempotents. Thus, by the corollary to Lemma $3.9, K$ is generated by $K \backslash K a$. Hence $K$ is finitely generated.

We can use Theorems 3.6 and 3.12 to give a complete characterisation of finitely generated cancellative archimedean semigroups. Those with idempotents are just finitely generated groups; those without idempotents are described in Theorem 3.13.

THEOREM 3.13. Let $S$ be a cancellative archimedean semigroup that has no idempotents. Then the following are equivalent:

(i) $S$ obeys the A.C.C.;

(ii) $S$ is finitely generated;

(iii) $S$ is isomorphic to a subsemigroup of $\mathbf{Z}^{+} \times G$ for some finite group $G$.

Proof. Clearly (i) implies (ii). If $S$ is finitely generated, then, by Proposition 2.7, $S$ is isomorphic to a subsemigroup of $S / \tau \times S / \rho_{a}$ for each $a \in S$, where, by the corollary to Theorem $3.5, S / \tau$ is isomorphic to a subsemigroup of $\mathrm{Z}^{+}$. Further $G=S / \rho_{a}$ is a finitely generated rational group and is therefore finite. Hence (ii) implies (iii).

On the other hand, $\mathrm{Z}^{+} \times G$ is finitely generated when $G$ is a finite group and is cancellative and archimedean without idempotents. Hence, by Theorem 3.12, $Z^{+} \times G$ obeys the A.C.C. Consequently, if $S$ is isomorphic to a subsemigroup of $\mathbf{Z}^{+} \times G, S$ also obeys the A.C.C.

Remark. That (ii) implies (iii) in Theorem 3.13, has also been shown by Higgins and Tamura (see the appendix to [11]). Their method however is different from ours.

As a corollary to Theorem 3.13, we can essentially obtain the results of Petrich [7] on cancellative archimedean semigroups with two generators and no idempotents.

THEOREM 3.14. Let $S$ be a cancellative rational semigroup with two generators and no idempotents. Then $S$ is isomorphic to a semigroup $C(n, m)$ constructed as follows:

Let $m, n$ be positive integers with $n \leqq m$; then $C(n, m)$ is the semigroup of all complex numbers of the form

$$
2^{\lambda / n+\mu / m} e^{2 \pi i \mu / m},
$$

where $\lambda, \mu$ are non-negative integers, not both zero. 
Proof. By the corollary to Theorem 3.5, we may suppose that $S / \tau \subseteq \mathbf{Z}^{+}$; let $a, b$ be generators for $S$ such that $a \tau^{\natural} \geqq b \tau^{\natural}$. Further, let $n$ and $m$ be the least positive integers $r$ and $s$, respectively, such that $a^{r}=b^{s}$. Then $m$ is the order of the cyclic group generated by $b \rho_{a}^{\natural}$ and $n . a \tau^{\natural}=m . b \tau^{\natural}$; the latter implies that $n \leqq m$. We may thus regard $S$ as the subsemigroup of $\mathbf{Z}^{+} \times G$ generated by $\left(a \tau^{\natural}, e\right)$ and $\left(b \tau^{\natural}, b \rho_{a}^{\natural}\right)$, where $e$ is the identity of $G$. The mapping $S \rightarrow C(n, m)$ generated by

$$
\left(a \tau^{\natural}, e\right) \rightarrow 2^{1 / n}, \quad\left(b \tau^{\natural}, b \rho_{a}^{\natural}\right) \rightarrow 2^{1 / m} e^{2 \pi i / m}
$$

is clearly an isomorphism. Hence we have the result.

Theorem 3.13 shows that a finitely generated cancellative rational semigroup $S$ without idempotents can be embedded in $\mathrm{Z}^{+} \times F$ for some finite group $F$. However it is easy to see that $S$ need not be isomorphic to $Z^{+} \times G$ for any finite group $G$. We end this section by giving necessary and sufficient conditions on $S$ in order that $S \approx \mathbf{Z}^{+} \times G$ for some finite group $G$.

We require the following simple group-theoretic lemma [4, page 80, Question $1(a)$ ].

LeMma 3.15. Let $G=\left\{a_{1}, \ldots, a_{n}\right\}$ be a finite group. Then $\left(a_{1} a_{2} \ldots a_{n}\right)^{2}=e$, where $e$ is the identity of $G$.

THEOREM 3.16. Let $S$ be a finitely generated cancellative semigroup without idempotents. Then $S \approx Z^{+} \times G$ for some finite group $G$ if and only if $S^{2}=S a$ for each $a \in S \backslash S^{2}$.

Proof. Let $X=\left\{a_{1}, \ldots, a_{n}\right\}$ be a minimal generating set for $S$ and suppose, for example, that $a_{1} \in S^{2}$. Then $a_{1}=a_{1}^{r_{1}} \ldots a_{n}^{r_{n}}$, where $r_{1}, \ldots, r_{n}$ are non-negative integers with $\sum r_{i} \geqq 2$. Since $S$ does not have an idempotent, $r_{1}$ must be zero; otherwise we should have $a_{1}=a_{1} x$ for some $x \in S$, which, because $S$ is cancellative, would imply that $x$ is an idempotent. Hence $a_{1}$ is a product of powers of $a_{2}, \ldots, a_{n}$. This contradicts the minimality of $X$; so we must have $X=S \mid S^{2}$.

Now suppose that $S^{2}=S a$ for each $a \in S \backslash S^{2}$. Then, in particular, $a_{i}^{2} \in S a_{j}$ for $1 \leqq i, j \leqq n$ so that the generators of $S$ are archimedean equivalent. Since each archimedean class of $S$ is a subsemigroup, it follows that $S$ is archimedean. Further, an easy induction shows that $S^{m}=S x$ for each $x=a_{1}^{s_{1}} \ldots a_{n}^{s_{n}}$, where $s_{1}, \ldots, s_{n}$ are non-negative integers such that $\sum s_{i}=$ $m-1 \geqq 1$. Also $x \in S^{m-1} \backslash S^{m}$, since otherwise $x \in S x$, which implies that $S$ has an idempotent.

Let $y=\left(a_{1} \ldots a_{n}\right)^{2}$ and pick $a \in S \backslash S^{2}$. Then $y \in S^{2 n}\left|S^{2 n+1}=S a^{2 n-1}\right| S^{2} a^{2 n-1}$; so $y=a^{*} a^{2 n-1}$, where $a^{*} \in S \backslash S^{2}$. Since $S$ is finitely generated and archimedean, $S / \rho_{a}$ is a finite group with identity $a \rho_{a}^{\natural}$ and it follows, from Lemma 3.15, that $(y, a) \in \rho_{a},\left(y, a^{*}\right) \in \rho_{a}$; thus $\left(a, a^{*}\right) \in \rho_{a}$. But distinct elements of $S \backslash S a=S \backslash S^{2}$ are distinct modulo $\rho_{a}$; so we must have $a^{*}=a$. Hence $y=a^{2 n}$ for each $a \in S \backslash S^{2}$, so that all the generators of $S$ are equivalent modulo $\tau$. It follows that we may assume that $S / \tau \subseteq \mathbf{Z}^{+}$and that $a \tau^{\natural}=1$.

By Theorem 3.13, $S$ is isomorphic to a subsemigroup of $S / \tau \times S / \rho_{a}$. Under this embedding, $a_{i}$ is mapped onto $\left(1, a_{i} \rho_{a}^{\natural}\right)$ for each $i=1, \ldots, n$. Since $\left(S \backslash S^{2}\right) \rho_{a}^{\natural}=S \rho_{a}^{\natural}$, it follows that the elements $\left(1, a_{i} \rho_{a}^{q}\right)$ generate $Z^{+} \times S / \rho_{a}$. Hence $S \approx Z^{+} \times S / \rho_{a}$.

Conversely, suppose that $S=\mathbf{Z}^{+} \times G$, where $G$ is a finite group. Then it is easy to see 
that $S \backslash S^{2}=\{(1, g): g \in G\}$. But, if $n \geqq 2$ is a positive integer and $f, g \in G$, then

$$
(n, f)=\left(n-1, f g^{-1}\right)(1, g) \text {. }
$$

Hence $S^{2}=S a$ for each $a=(1, g) \in S \mid S^{2}$.

4. Archimedean semigroups with idempotents. The following interesting theorem characterises finitely generated archimedean semigroups with idempotents. Note that an archimedean semigroup $S$ has an idempotent $e$ if and only if $\operatorname{Ker} S \neq \square$; in this case $\operatorname{Ker} S=S e$.

THEOREM 4.1. Let $S$ be a finitely generated archimedean semigroup. Then $\operatorname{Ker} S=$ $\bigcap\left\{S^{n}: n \geqq 1\right\}$ (where the possibility $\operatorname{Ker} S=\square$ is allowed).

Proof. If $\operatorname{Ker} S=\square$, then certainly $\operatorname{Ker} S \subseteq \bigcap\left\{S^{n}: n \geqq 1\right\}$; if $\operatorname{Ker} S \neq \square$, then $\operatorname{Ker} S$ is the minimum ideal of $S$ and again $\operatorname{Ker} S \subseteq \bigcap\left\{S^{n}: n \geqq 1\right\}$. Hence, in both cases, Ker $S \subseteq$ $\bigcap\left\{S^{n}: n \geqq 1\right\}$.

Conversely, if $\bigcap\left\{S^{n}: n \geqq 1\right\}=\square$, then $\bigcap\left\{S^{n}: n \geqq 1\right\} \subseteq \operatorname{Ker} S$. Thus we may suppose that $\bigcap\left\{S^{n}: n \geqq 1\right\} \neq \square$. Let $x$ be in this intersection and let $a_{1}, \ldots, a_{n}$ generate $S$. Then there exist $y_{i} \in S$ and $N \in \mathbf{Z}^{+}$such that $a_{i}^{N}=x y_{i}$ for $i=i, \ldots, n$. Since $x \in S^{N+1}, x=a_{j}^{N} z$ for some $z \in S$ and some $\mathrm{j} \leqq n$. This implies that $a_{j}^{N}=x y_{j}=a_{j}^{N} z y_{j}$ and therefore, by Proposition 2.4, Corollary $1, S$ has an idempotent $e$.

Since $S$ is archimedean, $e$ is the unique idempotent of $S$ and $\operatorname{Ker} S=S e$. Now $\left(z y_{j}\right)^{m} \in$ $\operatorname{Ker} S$ for some $m \in \mathbf{Z}^{+}$and so, since $a_{j}^{N}=a_{j}^{N}\left(z y_{j}\right)^{m}, a_{j}^{N} \in \operatorname{Ker} S$. Hence $x=a_{j}^{N} z \in \operatorname{Ker} S$. This shows that $\bigcap\left\{S^{n}: n \geqq 1\right\} \subseteq \operatorname{Ker} S$ and the result follows.

COROLLARY 1. Let $S$ be a finitely generated archimedean semigroup. Then $S$ has an idempotent if and only if $\bigcap\left\{S^{n}: n \geqq 1\right\} \neq \square$.

COROLLARY 2. Let $S$ be a finitely generated archimedean semigroup. Then $S$ is a group if and only if $S^{2}=S$.

Proof. If $S^{2}=S$ then, by Theorem 4.1, $S=\operatorname{Ker} S$. Hence $S$ is a group. The converse is immediate.

COROLlary. 3. Let $S$ be a finitely generated semigroup that satisfies $S^{2}=S$. Then $S$ contains idempotents.

Proof. Let $S_{\alpha}$ be a maximal archimedean component of $S$. Then $S_{\alpha}$ is finitely generated and $S_{\alpha}^{2}=S_{\alpha}$. By the previous corollary, $S_{\alpha}$ is a group and the result follows.

COROLGARY 4. If $S$ is a finitely generated cancellative semigroup, then $S$ has an identity if and only if $S^{2}=S$.

COROLlaRY 5. Let $S$ be a finitely generated separative semigroup whose maximal semilattice homomorphic image has no non-maximal meet irreducible elements. Then $S$ is a union of groups if and only if $S^{2}=S$.

Proof. Under the hypotheses of the corollary, the maximal semilattice homomorphic image of $S$ is generated by its maximal elements. Thus, if $S^{2}=S$, each archimedean com- 
ponent of $S$ contains an idempotent and so is a group. Thus, if $S^{2}=S, S$ is a union of groups. The converse is immediate.

The results of Theorem 4.1 and its corollaries are false without the assumption that $S$ is finitely generated. This is seen by considering the semigroup $\mathbf{Q}^{+}$of positive rationals.

Proposition 4.2. Let $S$ be an archimedean semigroup with an idempotent. Then $S$ is finitely generated if and only if $\operatorname{Ker} S$ is a finitely generated group and $S / \operatorname{Ker} S$ is a finite nilpotent semigroup.

Proof. Let $e$ be the unique idempotent of $S$. Then $\operatorname{Ker} S=S e$ is a group. Hence, if $S$ is finitely generated, $\operatorname{Ker} S$ is a finitely generated group and $S / \operatorname{Ker} S$, being finitely generated and nilpotent, is finite. The converse is immediate.

We can use Proposition 4.2 to characterise archimedean semigroups with idempotents that obey the A.C.C.

THEOREM 4.3. Let $S$ be an archimedean semigroup with an idempotent. Then $S$ obeys the A.C.C. if and only if $S / \operatorname{Ker} S$ is finite and $\operatorname{Ker} S$ is a finite group or is isomorphic to $\mathbf{Z} \times F$ for some finite group $F$.

Proof. If $S$ obeys the A.C.C., then, as in Proposition 4.2, $S / \operatorname{Ker} S$ is finite and nilpotent. Further, $\operatorname{Ker} S$ is a finitely generated group and so is isomorphic to $\mathbf{Z}^{n} \times F$, where $F$ is a finite group and $n$ is a non-negative integer. Now $\mathbf{Z}^{+} \times \mathbf{Z}^{+}$does not obey the A.C.C. while $\mathrm{Ker} S$ does; hence we must have $n \leqq 1$. Therefore either $\operatorname{Ker} S \approx F$ or $\operatorname{Ker} S \approx \mathbf{Z} \times F$.

Conversely, if $\operatorname{Ker} S$ is finite, then $S$ is finite and therefore obeys the A.C.C. On the other hand, suppose that $\operatorname{Ker} S \approx \mathbf{Z} \times F$, where $F$ is a finite group and let $K$ be a subsemigroup of $S$. Then

$$
K=\{K \cap S \mid \operatorname{Ker} S\} \cup\{K \cap\{0\} \times F\} \cup\left\{K \cap Z^{+} \times F\right\} \cup\left\{K \cap Z^{-} \times F\right\} .
$$

The first two factors in this expression are finite while $\mathrm{Z}^{+} \times F$ and $\mathrm{Z}^{-} \times F$ are isomorphic and obey the A.C.C., by Theorem 3.13. Hence $K$ is finitely generated. By Proposition 1.5, it follows that $S$ obeys the A.C.C.

REMARK. There is a certain parallel between the structure of finitely generated archimedean semigroups with idempotents and that of those without idempotents. By Proposition 4.2, the former type are ideal extensions of finitely generated groups by finite nilpotent semigroups. However, as noted after Theorem 3.10, the latter are ideal extensions of finitely generated rational cancellative semigroups by finite nilpotent semigroups.

Yamada [12] has given an inductive construction for finite nilpotent semigroups. Hence, by means of Theorem 3.10 and Proposition 4.2, one can, in principle, construct all finitely generated archimedean semigroups.

To complete this section, we prove a result that gives a partial answer to the question of when an archimedean semigroup with an idempotent can be embedded in a finitely generated semigroup.

Proposition 4.4. Let $S$ be an archimedean semigroup with an idempotent e. If $S \backslash S e$ is finite, then $S$ can be embedded in a finitely generated semigroup if and only if $S$ is finitely generated. 
Proof. Suppose that $S \subseteq C$, where $C$ is a finitely generated semigroup. Then $\operatorname{Ker} S$ is a subgroup of $C$ and so, by Proposition 1.4, is finitely generated. Since $S \backslash \operatorname{Ker} S=S \backslash S e$ is finite, it follows that $S$ is finitely generated.

The converse is clear.

The following example shows that the assumption that $S \mid S e$ is finite is necessary in Proposition 4.4.

EXAMPLE 4.5. Let $T=F\left(a, b, z: a z=b z=b^{2}=z\right)$ and let $S=\{b, z\} \cup b\langle a\rangle$. Then $S$ is an infinite semigroup with $S^{2}=\{z\}$; in particular $S$ is archimedean. Since $S \backslash S^{2}=S \backslash\{z\}$ is infinite, $S$ cannot be finitely generated. However $S$ is a subsemigroup of the finitely generated semigroup $T$.

5. Separative semigroups that obey the A.C.C. In this section, we shall apply the theory given in $\S 3$ and 4 to characterise those separative and cancellative semigroups that obey the A.C.C.

THEOREM 5.1. Let $S$ be a separative semigroup. Then $S$ obeys the A.C.C. if and only if $S$ can be embedded in a finite semilattice of groups $\mathbf{Z} \times F_{\alpha}$, where each $F_{\alpha}$ is a finite group.

Proof. If $S$ obeys the A.C.C., then, by Theorems 3.13 and 4.3, each archimedean component $S_{\alpha}$ is either isomorphic to $\mathbf{Z} \times F_{\alpha}$ or to $F_{\alpha}$ or is isomorphically embedded in $\mathrm{Z}^{+} \times F_{\alpha}$ for some finite group $F_{\alpha}$. Thus, whatever the case, $S_{\alpha}$ is isomorphic to a subsemigroup of $\mathrm{Z} \times F_{\alpha}$. It follows, from [1, Theorem 4.17], that $S$ can be embedded in the finite semilattice of groups $\mathbf{Z} \times F_{\alpha}$.

Conversely, if $S$ is embedded in a finite semilattice of groups $\mathbf{Z} \times F_{\alpha}$, then

$$
S=\bigcup\left\{S \cap \mathbf{Z} \times F_{\alpha}\right\},
$$

where this is a finite union. But, as in the proof of Theorem 4.3, each $\mathbf{Z} \times F_{\alpha}$ obeys the A.C.C. Hence $S$ obeys the A.C.C.

We shall use the following proposition in characterising cancellative semigroups that obey the A.C.C.; the proposition is however of interest in its own right.

Proposition 5.2. Let $S_{\alpha}$ and $S_{\beta}$ be distinct archimedean components of a cancellative semigroup $S$. If $a \in S_{\alpha}, b \in S_{\beta}$ have infinite order, then $\langle a, b\rangle \approx F(a, b)$.

Proof. Suppose that $a^{r} b^{s}=a^{u} b^{v}$, where $r, s, u, v$ are non-negative integers and $(r, s) \neq(u, v)$. Then, since $S$ is cancellative and $a, b$ have infinite order, $r \neq u, s \neq v$; so we may assume, without loss of generality, that $r>u$. The cancellativity of $S$ then gives $a^{r-u} b^{s-v}=1$ or $a^{r-u}=b^{v-s}$ according as $s>v$ or $s<v$. In the first case, $a$ and $b$ are invertible in $S$ and so are archimedean equivalent while, in the second case, it is clear that $a$ and $b$ are archimedean equivalent. This contradicts the fact that $S_{\alpha}$ and $S_{\beta}$ are distinct. Hence $a^{r} b^{s}=a^{u} b^{v}$ implies $r=u, s=v$ and so $\langle a, b\rangle \approx F(a, b)$.

COROLLARY. If $S$ is a cancellative semigroup that obeys the A.C.C., then $S$ has at most two archimedean components. 
Proof. Suppose that $S$ has three archimedean components (at least) $S_{\alpha}, S_{\beta}, S_{\gamma}$. Then, since a cancellative semigroup has at most one idempotent, at least two of $S_{\alpha}, S_{\beta}, S_{\gamma}$ contain elements of infinite order. It follows, from Proposition 5.2, that $S$ contains the free semigroup $F(a, b)$ on two generators as a subsemigroup. But, by Example 1.2, $F(a, b)$ does not obey the A.C.C. Hence $S$ does not obey the A.C.C.

THEOREM 5.3. Let $S$ be a cancellative semigroup. Then $S$ obeys the A.C.C. if and only if $S$ can be embedded in $\mathrm{Z} \times G$ for some finite group $G$.

Proof. Suppose that $S$ obeys the A.C.C. Then, by the corollary to Proposition 5.2, $S$ has at most two archimedean components. If $S$ is archimedean, then, by Theorems 3.13 and 4.3, $S$ can be embedded in $\mathbf{Z} \times G$ for some finite group $G$.

If $S$ has two archimedean components, let these be $S_{\alpha}, S_{\beta}$, where $\alpha<\beta$. Since a cancellative semigroup has at most one idempotent, each element of $S_{\alpha}$ has infinite order. If $S_{\beta}$ contained an element of infinite order, then, by Proposition 5.2, $S$ would contain the free semigroup on two generators as a subsemigroup and so would not obey the A.C.C. Hence $S_{\beta}$ is periodic and, since it is finitely generated, in fact, a finite group.

By Theorem 3.13, $S_{\alpha}$ can be embedded in $Z^{+} \times G$ for some finite group $G$ and, without loss of generality, we can assume that $\mathbf{Z} \times G$ is the group of quotients of $S_{\alpha}$. Then $T=S_{\beta} \cup \mathbf{Z} \times G$ is the union of groups in which $S$ is naturally embedded [1, Theorem 4.17]. Since $S_{\beta}$ is finite, it follows from [1, Theorem 4.11] that multiplication in $T$ is defined by

$$
x y=\left\{\begin{array}{lll}
f h & \text { if } \quad x=f, y=h \in S_{\beta}, \\
(n, f \theta g) & \text { if } \quad x=f \in S_{\beta}, y=(n, g) \in \mathbf{Z} \times G, \\
(m+n, g h) & \text { if } \quad x=(m, g), y=(n, h) \in \mathbf{Z} \times G,
\end{array}\right.
$$

where $\theta$ is a homomorphism of $S_{\beta}$ into $G$. Suppose that $f \in \operatorname{Ker} \theta$ and let $(n, g) \in S_{\beta}$. Then

$$
f \cdot(n, g)=(n, f \theta g)=(n, g)=e \cdot(n, g),
$$

where $e$ is the identity of $S_{\beta}$. Hence, since $S$ is cancellative, $f=e$. Thus $\theta$ is one-to-one and it is straightforward to verify that $\varphi: S \rightarrow \mathbf{Z} \times G$ defined by

$$
x \varphi=\left\{\begin{array}{ccc}
(0, x \theta) & \text { if } & x \in S_{\beta}, \\
x & \text { if } & x \in S_{\alpha},
\end{array}\right.
$$

is an embedding of $S$ into $\mathbf{Z} \times G$.

Conversely, as in the proof of Theorem $4.3, \mathrm{Z} \times G$ obeys the A.C.C. Hence each subsemigroup of $\mathbf{Z} \times G$ obeys the A.C.C.

As a final application of the theory in $\$ \S 3$ and 4 , we shall determine the structure of all two-generator cancellative semigroups.

THEOREM 5.4. Let $S$ be a cancellative semigroup with two generators. Then $S$ is isomorphic to exactly one of the following semigroups.

(1) $\mathbf{Z}$;

(2) $(\mathbf{Z} / m \mathbf{Z}) \times(\mathbf{Z} / n \mathbf{Z})$ for some non-negative integers $m, n$ with $n \geqq 2$; 
(3) $C(n, m)$ for some positive integers $m, n$ with $n \leqq m$ and $(n, m) \neq(1,1)$;

(4) $\mathbf{N} \times(\mathbf{Z} / m \mathbf{Z})$ for some positive integer $m$, where $\mathbf{N}$ denotes the additive semigroup of non-negative integers;

(5) $F(a, b)$, the free semigroup on two generators $a, b$.

Conversely, each of these semigroups is cancellative and has two generators.

Proof. If $S$ is archimedean, then, by Theorem 3.16, either $S \approx C(n, m)$ for some $m, n$ or else $S$ is a group which is generated as a semigroup by two elements. But it is easy to see that such a group is isomorphic to $\mathbf{Z}$ or to $(\mathbf{Z} / m \mathbf{Z}) \times(\mathbf{Z} / n \mathbf{Z})$ for some $m, n$.

If $S$ is not archimedean, let $a, b$ be generators for $S$ and let $S_{\alpha}, S_{\beta}$ be the archimedean components containing $a$ and $b$ respectively; suppose, for example, that $\alpha \neq \beta$. Then $S_{\alpha}$ is a maximal archimedean component of $S$ and so is generated by $a$; thus $S_{\alpha}$ is cyclic. Further, since only the maximum archimedean class of a cancellative semigroup can contain an idempotent, each element of $S_{\beta}$, in particular $b$, has infinite order.

If $a$ has infinite order, then, by Proposition 5.2, $S=\langle a, b\rangle \approx F(a, b)$. If $a$ does not have infinite order, then $S_{\alpha}=\langle a\rangle$ is a cyclic group and so $S_{\alpha} \approx \mathbf{Z} / m \mathbf{Z}$ for some positive integer $m$. Further, each element of $S$ can be uniquely written as $a^{n} b^{n}$, where $r, n$ are non-negative integers with $0 \leqq r<m$; an argument like that used in the proof of Proposition 5.2 shows the uniqueness of the elements. Hence $S \approx \mathrm{N} \times(\mathrm{Z} / m \mathrm{Z})$.

The converse is straightforward.

COROLlaRY. A cancellative semigroup with two generators is free or obeys the A.C.C.

\section{Extending characters on finitely generated semigroups.}

Definition 6.1. [6] A semigroup $S$ is said to have the character extension property if each character of each subsemigroup of $S$ can be extended to a character of $S$; by a character of $S$ we mean a homomorphism of $S$ into the multiplicative semigroup of complex numbers.

In this section we shall use the structure theory obtained so far to characterise finitely generated semigroups that have the character extension property. In [6] it is shown that a semigroup has the character extension property if and only if each archimedean component is rational. The following theorem characterises the cancellative rational subsemigroups of finitely generated semigroups.

THEOREM 6.2. Let $S$ be a cancellative rational semigroup without idempotents. Then $S$ can be embedded in a finitely generated semigroup if and only if $S$ is finitely generated.

Proof. Suppose that $S \subseteq C$, where $S$ is a cancellative rational semigroup and $C$ is finitely generated. Then, since $S$ is rational, $S \subseteq C_{\alpha}$ for some archimedean component $C_{\alpha}$ of $C$. Let $D=\bigcup\left\{C_{\beta}: \beta \geqq \alpha\right\}$, where $C=\bigcup\left\{C_{\lambda}: \lambda \in \Lambda\right\}$ is the expression of $C$ as the union of a semilattice of archimedean semigroups, and pick $a \in S$. It is clear that $D$ has minimum archimedean component $C_{\alpha}$ so that, by the corollary to Proposition $2.6, G=D / \rho_{\mathrm{a}}$ is a group.

Define a mapping $\theta: S \rightarrow G^{0}$ as follows:

$$
x \theta=\left\{\begin{array}{ccl}
(x a) \rho_{a}^{\natural} & \text { if } & x \in D, \\
0 & \text { if } & x \in S \backslash D .
\end{array}\right.
$$

$\mathbf{E}$ 
Since $D$ is the complement of a prime ideal of $C\left[6\right.$, Lemma 1.3] and since $a \rho_{a}^{\natural}$ is the identity of $G$, it is easy to see that $\theta$ is a homomorphism of $C$ onto $G^{0}$. Hence $G^{0}$ is finitely generated and it follows from Proposition 1.4 that $G$ is finitely generated. Since $S$ is rational and contains $a, S \theta$ is a periodic subsemigroup of $G$. Hence it follows that $S \theta$ is a finite subgroup of $G$.

Suppose that $x, y \in S \backslash S a$ are such that $x \theta=y \theta$; then, for some $m, n \in \mathbf{Z}^{+}, x a^{m}=y a^{n}$. But, since $S$ is cancellative, and $x, y \in S \mid S a$, this means that $x=y$. Thus distinct elements of $S \mid S a$ are mapped onto distinct elements of $S$; since the latter is finite, this shows that $S \mid S a$ is finite. By the corollary to Lemma 3.9, it follows that $S$ is finitely generated.

COROLLARY. $\mathbf{Q}^{+}$cannot be embedded in a finitely generated semigroup.

If $S$ is a semigroup without idempotents, then both the other hyoptheses of Theorem 6.2 are necessary in order that the conclusions of the theorem should hold. This is shown by the following examples.

Example 6.3. Let $K$ be as in Example 1.2. Then $K$ is cancellative but not rational or finitely generated. However $K \subseteq F(a, b)$.

EXAMPLE 6.4. Let $K$ be as in Example 1.3. Then $K$ is rational but not cancellative or finitely generated; yet $K \subseteq F\left(a, b ; a^{2} b^{2}=b^{2}\right)$.

THEOREM 6.5. Let $S$ be a finitely generated separative semigroup. Then $S$ has the character extension property if and only if each archimedean component is a finite group or can be embedded in $\mathbf{Z}^{+} \times G$ for some finite group $G$.

Proof. If each archimedean component is a finite group or can be embedded in $\mathbf{Z}^{+} \times G$ for some finite group $G$, then each archimedean component is rational. Hence, by [6, Theorem 3.5], $S$ has the character extension property.

Conversely, suppose that $S$ has the character extension property and let $S_{\alpha}$ be an archimedean component of $S$. If $S_{\alpha}$ has an idempotent, $S_{\alpha}$ is a finitely generated rational group and is therefore finite. If $S_{\alpha}$ does not have an idempotent, then, by Theorem 6.2, $S_{\alpha}$ satisfies the hypotheses of Theorem 3.13. Hence $S_{\alpha}$ can be embedded in $Z^{+} \times G$ for some finite group $G$.

COROLLARY 1. Let $S$ be a finitely generated separative semigroup. Then $S$ has the character extension property if and only if each maximal subgroup is finite and S obeys the A.C.C.

COROLLARY 2. Let $S$ be a finitely generated cancellative semigroup. Then $S$ has the character extension property if and only if $S$ can be embedded in $\mathrm{N} \times G$ for some finite group $G$.

Proof. Suppose that $S$ has the character extension property. Then, by Corollary $1, S$ obeys the A.C.C. and has finite or trivial group of units. The proof of Theorem 5.3 shows that, under these conditions, $S$ can be embedded in $\mathbf{N} \times G$ for some finite group $G$.

The converse is immediate from Theorem 6.5.

\section{REFERENCES}

1. A. H. Clifford and G. B. Preston, The algebraic theory of semigroups, Vol. I, Math. Surveys of the American Math. Soc. 7 (Providence, R.I., 1961). 
2. P. Dubreil, Contribution a la theorie des demigroupes. Mem. Acad. Sci. Inst. France (2) 63, no. 3 (1941), 1-52.

3. L. Fuchs, Abelian groups (London, 1960).

4. I. N. Herstein, Topics in algebra (Waltham, Mass., 1964). 365-371.

5. R. G. Levin, On commutative nonpotent archimedean semigroups, Pacific J. Math, 27 (1968),

6. D. B. McAlister, Characters on commutative semigroups, Quarterly J. Math. Oxford Ser. 2, 19 (1968) 141-157.

7. M. Petrich, On the structure of a class of commutative semigroups, Czechoslovak Math. J. 14 (1964), 147-153.

8. L. Redei, The theory of finitely generated commutative semigroups (London, 1965).

9. T. Tamura, Commutative nonpotent archimedean semigroup with cancellation law, Gakugei Tokoshima Univ. 8 (1957), 5-11.

10. T. Tamura, Notes on commutative archimedean semigroups, I, II, Proc. Japan Acad. 42 (1966) $35-40,545-548$.

11. T. Tamura, Construction of trees and commutative archimedean semigroups, Math. Nachr. 36 (1968), 257-287. 94-98.

12. M. Yamada, Construction of finite commutative $z$-semigroups, Proc. Japan Acad. 40 (1964),

NORTHERN ILLINOIS UNIVERSITY

De Kalb, Illinois 60115, U.S.A. 